INTERNATIONAL JOURNAL
PHARMACEUTICAL SCIENCES
RESEARCH
RES

Received on 06 December, 2017; received in revised form, 14 February, 2018; accepted, 04 March, 2018; published 01 August, 2018

\title{
DESIGN OF CONTROLLED RELEASE NON-ERODIBLE POLYMERIC ATENOLOL MATRIX TABLET USING MICROWAVE OVEN-ASSISTED SINTERING TECHNIQUE
}

\author{
Satish Polshettiwar *1,2 and Rahul Hajare ${ }^{2}$
}

Department of Pharmaceutics ${ }^{1}$, MAEERS, Maharashtra Institute of Pharmacy, MIT Campus, Kothrud, Pune - 411038, Maharashtra, India.

Centre for Research and Development ${ }^{2}$, Pacific University, Udaipur - 313003, Rajasthan, India.

\section{Keywords:}

Atenolol, Controlled

release systems, Preformulation,

Sintering, Eudragit S-100

\section{Correspondence to Author:}

Satish Polshettiwar

Department of Pharmaceutics, Maharashtra Institute of Pharmacy, Kothrud, Pune - 411038,

Maharashtra, India.

E-mail: satishmip@gmail.com

\begin{abstract}
The objective of the present study was to evaluate the effect of microwave sintering condition on matrix formation and subsequent drug release from polymer matrix tablet for controlled release. The atenolol floating SR matrix tablets were prepared by using tragacanth, sodium bicarbonate, Eudragit S-100 and citric acid was incorporated as a gas generating agents. Atenolol controlled release tablets were estimated in terms of their physico-chemical parameter like weight variation, friability, hardness, drug content, contact angle, floating lag time in-vitro drug release, specific gravity and stability study as per ICH guidelines. The results of in-vitro drug release studies showed that the optimized formulation (F6, L2, I2, H4) could extend drug release (99.5\%) for 24 hours. The release pattern of atenolol was fitted to different models based on coefficient of correlation (r). The present work also deals with the application of sintering technique to matrix tablets of atenolol to achieve sustained release. The results of the present work stated that sintering caused decrease in drug release as compared to unsintered tablets. It was found that F6, L2, I2 and H\$ gave better results compared to other formulations. The stability studies were carried out according to ICH guideline which indicates that the selected formulations were stable. Hence we finally conclude that microwave oven sintering is better than conventional hot air oven sintering process in preparation of controlled release tablets.
\end{abstract}

INTRODUCTION: Controlled release drug delivery has become the norm in dosage form design and intensive research has been undertaken in achieving better drug product effectiveness reliability and safety. The objective of the design and manufacture of the compressed CR tablet is to deliver orally the correct amount of drug in the desired location and to have its chemical integrity protected to the point.

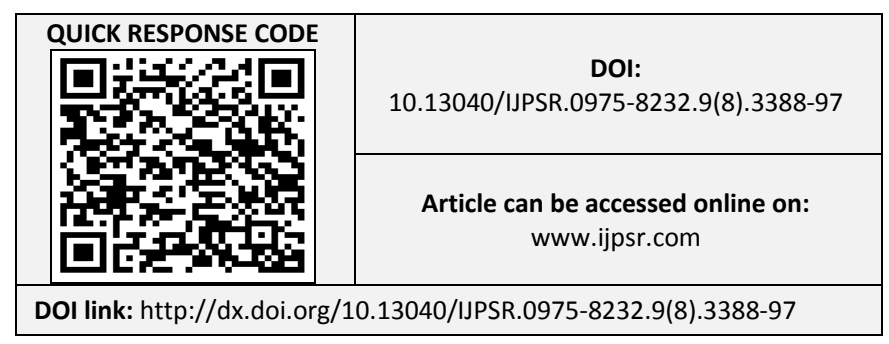

Recent advances in Novel Drug Delivery System (NDDS) aims to enhance safety and efficacy of drug molecule by formulating a convenient dosage form for administration and to achieve better patient compliance ${ }^{1-3}$. Atenolol is $\beta-1$ cardio selective adrenergic receptor blocker, widely used in the treatment of hypertension. The drug is soluble in water (BCS Class III) and has half-life of $6-8 \mathrm{~h}$ with oral bioavailability of $50 \%$ due to smaller dose of drug (less than $50 \mathrm{mg}$ ).

In this study, an attempt was made to design and formulate the floating matrix tablets of atenolol so as to increase its gastric retention thereby ensuring slower and complete release of atenolol. The sustained drug delivery can be achieved by matrix tablet where a solid drug is dispersed in an 
insoluble matrix. The rate of release of drug is dependent on the rate of drug diffusion and not on the rate of solid diffusion. The matrix tablets can be prepared by wet granulation method. Wet granulation has several advantages over dry granulation method like better flow properties of granules and less generation of dust while mixing ${ }^{4}$ $-5,18$. Microwave sintering involves the heating of compact at a temperature below the melting point of the solid constituents in a controlled environment. It is simple, effective and economical process to obtain sustained release of drug from the tablet with comparatively less quantity of polymer ${ }^{6-7}$.

Present research work has been undertaken to evaluate the effect of sintering condition on matrix formation and subsequent drug release from polymer matrix tablet for controlled release. The present study highlights the use of a microwave oven for the sintering process in order to achieve more uniform heat distribution with reduction in time required for sintering. Administration of conventional tablets of atenolol has been reported to exhibit fluctuations in plasma drug levels, resulting either in manifestation of side effects or reduction in drug concentration at the receptor sites ${ }^{8,10}$.

MATERIALS AND METHODS: Materials: Atenolol (Yarrow Chem product, Mumbai), All other excipients was obtained from Eisen Pharmaceuticals, Pune.

\section{Methods:}

Preliminary Studies: Matrix granules of atenolol were prepared by wet granulation method. Various polymers such as ES - 100, tragacanth, ethyl cellulose, lactose, were investigated as singly and in combination in different ratios. Tragacanth was used as a binder to prepare granules, which were passed through sieve no. 16. The granules were dried at $45^{\circ} \mathrm{C}$ for $30 \mathrm{~min}$. The dried granules were screened through sieve no 16 and stored at ambient temperature for further studies. The granules were mixed with magnesium stearate and talc in require quantity and compressed using rotary tablet compression machine (Rimek) using $13 \mathrm{~mm}$ diameter flat punches. The tablets were placed in beaker and subjected to microwave irradiation at 450, 560 and 700 watt for 20,15 and $10 \mathrm{~min}$ in microwave oven.

Experimental Design: ${ }^{8}-10$ A $3^{2}$ full factorial design (Design Expert, Version 7, Stat-Ease Inc, Minneapolis, MN) was used to design the formulation. Dependent variable X1: Eudragit S 100 X2: Tragacanth and Independent Variables Y1. Drug Content Y2: Swelling Index Y3. Buoyancy was taken. Following batches were developed.

\section{RESULTS:}

TABLE 1: TABLET COMPOSITION OF ATENOLOL SUSTAINED RELEASE TABLETS (F1 TO F9)

\begin{tabular}{cccccccccc}
\hline Ingredient weight in mg & F1 & F2 & F3 & F4 & F5 & F6 & F7 & F8 & F9 \\
\hline Atenolol & 111 & 111 & 111 & 111 & 111 & 111 & 111 & 111 & 111 \\
Tragacanth & 30 & 30 & 30 & 40 & 40 & 40 & 50 & 50 & 50 \\
Eudragit S100 & 30 & 40 & 50 & 30 & 40 & 50 & 30 & 40 & 50 \\
Lactose & 10 & 10 & 10 & 10 & 10 & 10 & 10 & 10 & 10 \\
NaHCO $_{3}$ & 11 & 11 & 11 & 11 & 11 & 11 & 11 & 11 & 11 \\
Citric acid & 11 & 11 & 11 & 11 & 11 & 11 & 11 & 11 & 11 \\
Magnesium Stearate & 22 & 22 & 22 & 22 & 22 & 22 & 22 & 22 & 22 \\
MCC & 10 & 30 & 20 & 30 & 20 & 10 & 20 & 10 & 30 \\
Talc & 5 & 5 & 5 & 5 & 5 & 5 & 5 & 5 & 5 \\
Final weight & 270 & 270 & 270 & 270 & 270 & 270 & 270 & 270 & 270 \\
\hline
\end{tabular}

TABLE 2: TABLET COMPOSITION OF ATENOLOL SUSTAINED RELEASE TABLETS (L1 TO L9)

\begin{tabular}{cccccccccc}
\hline Ingredients weight in mg & L1 & L2 & L3 & L4 & L5 & L6 & L7 & L8 & L9 \\
\hline Atenolol & 111 & 111 & 111 & 111 & 111 & 111 & 111 & 111 & 111 \\
Tragaecanth & 30 & 30 & 30 & 30 & 30 & 30 & 30 & 30 & 30 \\
Eudragit L100 & 30 & 30 & 30 & 30 & 30 & 30 & 30 & 30 & 30 \\
Lactose & 10 & 10 & 10 & 10 & 10 & 10 & 10 & 10 \\
NaHCO & 11 & 11 & 11 & 11 & 11 & 11 & 11 & 11 \\
Citric acid & 11 & 11 & 11 & 11 & 11 & 11 & 11 & 11 \\
Magnesium Stearate & 22 & 22 & 22 & 22 & 22 & 22 & 22 & 22 \\
MCC & 10 & 10 & 10 & 10 & 10 & 10 & 10 & 10 \\
Talc & 5 & 5 & 5 & 5 & 5 & 5 & 5 & 5 & 5 \\
Final weight & 270 & 270 & 270 & 270 & 270 & 270 & 270 & 270 & 270 \\
Sintering temp $\left({ }^{0} \mathrm{C}\right)$ & 60 & 60 & 60 & 70 & 70 & 70 & 80 & 80 & 80 \\
Sintering time (mins) & 10 & 15 & 20 & 10 & 215 & 20 & 10 & 15 & 20 \\
\hline
\end{tabular}


TABLE 3: TABLET COMPOSITION OF ATENOLOL SUSTAINED RELEASE TABLETS (I1 TO I9)

\begin{tabular}{|c|c|c|c|c|c|c|c|c|c|}
\hline Ingredients weight in mg & I1 & I2 & I3 & I4 & I5 & I6 & I7 & I8 & I9 \\
\hline Atenolol & 111 & 111 & 111 & 111 & 111 & 111 & 111 & 111 & 111 \\
\hline Tragacanth & 40 & 40 & 40 & 40 & 40 & 40 & 40 & 40 & 40 \\
\hline Eudragit S100 & 40 & 40 & 40 & 40 & 40 & 40 & 40 & 40 & 40 \\
\hline Lactose & 10 & 10 & 10 & 10 & 10 & 10 & 10 & 10 & 10 \\
\hline $\mathrm{NaHCO}_{3}$ & 11 & 11 & 11 & 11 & 11 & 11 & 11 & 11 & 11 \\
\hline Citric acid & 11 & 11 & 11 & 11 & 11 & 11 & 11 & 11 & 11 \\
\hline Magnesium stearate & 22 & 22 & 22 & 22 & 22 & 22 & 22 & 22 & 22 \\
\hline $\mathrm{MCC}$ & 20 & 20 & 20 & 20 & 20 & 20 & 20 & 20 & 20 \\
\hline Talc & 5 & 5 & 5 & 5 & 5 & 5 & 5 & 5 & 5 \\
\hline Final weight & 270 & 270 & 270 & 270 & 270 & 270 & 270 & 270 & 270 \\
\hline Sintering temp $\left({ }^{\circ} \mathrm{C}\right)$ & $60{ }^{\circ} \mathrm{C}$ & $60{ }^{\circ} \mathrm{C}$ & $60{ }^{\circ} \mathrm{C}$ & $70^{\circ} \mathrm{C}$ & $70^{\circ} \mathrm{C}$ & $70^{\circ} \mathrm{C}$ & $80^{\circ} \mathrm{C}$ & $80^{\circ} \mathrm{C}$ & $80{ }^{\circ} \mathrm{C}$ \\
\hline Sintering time $(\mathrm{h})$ & 10 & 15 & 20 & 10 & 15 & 20 & 10 & 15 & 20 \\
\hline
\end{tabular}

TABLE 4: TABLET COMPOSITION OF ATENOLOL SUSTAINED RELEASE TABLETS (H1 TO H9)

\begin{tabular}{cccccccccc}
\hline Ingredients weight in mg & H1 & H2 & H3 & H4 & H5 & H6 & H7 & H8 & H9 \\
\hline Atenolol & 111 & 111 & 111 & 111 & 111 & 111 & 111 & 111 & 111 \\
Tragaecanth & 50 & 50 & 50 & 50 & 50 & 50 & 50 & 50 & 50 \\
Eudragit L100 & 50 & 50 & 50 & 50 & 50 & 50 & 50 & 50 & 50 \\
Lactose & 10 & 10 & 10 & 10 & 10 & 10 & 10 & 10 & 10 \\
NaHCO & 11 & 11 & 11 & 11 & 11 & 11 & 11 & 11 & 11 \\
Citric acid & 11 & 11 & 11 & 11 & 11 & 11 & 11 & 11 & 11 \\
Magnesium stearate & 22 & 22 & 22 & 22 & 22 & 22 & 22 & 22 & 22 \\
MCC & 30 & 30 & 30 & 30 & 30 & 30 & 30 & 30 & 30 \\
Talc & 5 & 5 & 5 & 5 & 5 & 5 & 5 & 5 & 5 \\
Final weight & 270 & 270 & 270 & 270 & 270 & 270 & 270 & 270 & 270 \\
Sintering temp $\left({ }^{\circ} \mathrm{C}\right)$ & $60^{\circ} \mathrm{C}$ & $60^{\circ} \mathrm{C}$ & $60{ }^{\circ} \mathrm{C}$ & $70^{\circ} \mathrm{C}$ & $70^{\circ} \mathrm{C}$ & $70^{\circ} \mathrm{C}$ & $80^{\circ} \mathrm{C}$ & $80^{\circ} \mathrm{C}$ & $80^{\circ} \mathrm{C}$ \\
Sintering time (min) & 10 & 15 & 20 & 10 & 15 & 20 & 10 & 15 & 20 \\
\hline
\end{tabular}

TABLE 5: DATA FOR BLEND EVALUATION OF FORMULATION (F1 TO F9)

\begin{tabular}{ccccc}
\hline Formulation no. & Angle of repose $\left.\mathbf{(}^{\mathbf{0}}\right)$ & $\mathbf{L B D}\left(\mathbf{g} / \mathbf{c m}^{\mathbf{2}}\right)$ & TBD $\mathbf{( g / \mathbf { c m } ^ { 2 } )}$ & Compressibility index $\mathbf{\%})$ \\
\hline F1 & $29^{0} 84^{\prime}$ & 0.3846 & 0.4166 & 7.68 \\
F2 & $22^{0} 39^{\prime}$ & 0.4545 & 0.5555 & 18.18 \\
F3 & $29^{0} 53^{\prime}$ & 0.4 & 0.5 & 20 \\
F4 & $26^{0} 90^{\prime}$ & 0.3846 & 0.4545 & 15.37 \\
F5 & $27^{0} 56^{\prime}$ & 0.3571 & 0.4132 & 13.57 \\
F6 & $25^{0} 93^{\prime}$ & 0.3125 & 0.3676 & 14.98 \\
F7 & $29^{0} 89^{\prime}$ & 0.3703 & 0.4504 & 17.78 \\
F8 & $28^{0} 75^{\prime}$ & 0.4201 & 0.5102 & 17.65 \\
F9 & $25^{0} 07^{\prime}$ & 0.3496 & 0.4065 & 13.99 \\
\hline
\end{tabular}

TABLE 6: DATA FOR BLEND EVALUATION OF FORMULATION (L1 TO L9)

\begin{tabular}{|c|c|c|c|c|}
\hline Formulation no. & Angle of repose $\left(^{0}\right)$ & $\operatorname{LBD}\left(\mathrm{g} / \mathrm{cm}^{2}\right)$ & $\operatorname{TBD}\left(\mathrm{g} / \mathrm{cm}^{2}\right)$ & Compressibility index (\%) \\
\hline L1 & 20.56 & 0.4576 & 0.5642 & 18.8940 \\
\hline L2 & 24.58 & 0.4892 & 0.5234 & 6.53419 \\
\hline L3 & 23.16 & $0 . .4615$ & 0.5846 & 21.0571 \\
\hline L4 & 26.57 & 0.5032 & 0.5948 & 15.4001 \\
\hline L5 & 25.34 & 0.4542 & 0.5346 & 15.0392 \\
\hline L6 & 27.65 & 0.3987 & 0.476 & 18.2321 \\
\hline L7 & 23.68 & 0.5542 & 0.6243 & 11.2285 \\
\hline L8 & 24.97 & 0.4653 & 0.5612 & 17.0883 \\
\hline L9 & 25.67 & 0.4565 & 0.5346 & 14.6090 \\
\hline
\end{tabular}

TABLE 7: DATA FOR BLEND EVALUATION OF FORMULATION (I1 TO I9)

\begin{tabular}{ccccc}
\hline Formulation no. & Angle of repose $\left.\mathbf{(}^{\mathbf{0}}\right)$ & LBD $\left(\mathbf{g} / \mathbf{c m}^{\mathbf{2}}\right)$ & TBD $\mathbf{( g / \mathbf { c m } ^ { 2 } )}$ & Compressibility index $(\boldsymbol{\%})$ \\
\hline I1 & 24.05 & 0.4576 & 0.5678 & 19.4082 \\
I2 & 23.48 & 0.5123 & 0.6743 & 24.02449 \\
I3 & 25.61 & 0.4861 & 0.6021 & 19.2659 \\
I4 & 27.08 & 0.5721 & 0.6431 & 11.0402 \\
I5 & 21. & 0.5143 & 0.5976 & 13.9390 \\
I6 & 26. & 0.4698 & 0.5124 & 8.31381 \\
I7 & 28. & 0.4137 & 0.5064 & 18.3056 \\
I8 & 30. & 0.5678 & 0.6542 & 13.2069 \\
I9 & 27. & 0.5490 & 0.6341 & 13.4206 \\
\hline
\end{tabular}


TABLE 8: DATA FOR BLEND EVALUATION OF FORMULATION (H1 TO H9)

\begin{tabular}{ccccc}
\hline Formulation no. & Angle of repose $\left.\mathbf{(}^{\mathbf{0}}\right)$ & LBD $\left(\mathbf{g} / \mathbf{c m}^{\mathbf{2}}\right)$ & TBD $\mathbf{( g / \mathbf { c m } ^ { 2 } )}$ & Compressibility index (\%) \\
\hline H1 & 30.14 & 0.4865 & 0.5472 & 11.09282 \\
H2 & 21.45 & 0.4216 & 0.5684 & 24.2688 \\
H3 & 27.08 & 0.4675 & 0.5824 & 19.7287 \\
H4 & 26.48 & 0.5032 & 0.6542 & 23.0816 \\
H5 & 28.46 & 0.5431 & 0.7102 & 23.5288 \\
H6 & 29.46 & 0.4862 & 0.5216 & 6.7861 \\
H7 & 24.15 & 0.4675 & 0.5462 & 14.4084 \\
H8 & 27.64 & 0.4642 & 0.5231 & 11.2598 \\
H9 & 23.08 & 0.5548 & 0.6742 & 17.7098 \\
\hline
\end{tabular}

Post Compression Parameters:

TABLE 9: PHYSICAL PROPERTIES OF TABLET FORMULATIONS (F1 TO F9) OF ATENOLOL SUSTAINED RELEASE MATRIX TABLETS

\begin{tabular}{|c|c|c|c|c|c|c|c|c|c|}
\hline \multirow[t]{2}{*}{ Parameters } & \multicolumn{9}{|c|}{ Formulation Codes } \\
\hline & F1 & F2 & F3 & F4 & F5 & F6 & F7 & F8 & F9 \\
\hline Drug content $(\%)$ & 99.53 & 97.09 & 96.51 & 101.16 & 98.02 & 101.86 & 99.18 & 102.32 & 101.86 \\
\hline Hardness $\left(\mathrm{kg} / \mathrm{cm}^{3}\right)$ & 8.6 & 9.6 & 8.8 & 11.2 & 10.4 & 8.9 & 9.8 & 10.4 & 9.8 \\
\hline Swelling index (\%) & 193.24 & 203.01 & 178.32 & 181.39 & 206.21 & 221.25 & 185.67 & 206.17 & 222.05 \\
\hline Buoyancy lag time (min) & 2.15 & 2 & No float & 2 & 1.30 & 1 & 1.30 & 0.45 & 0.55 \\
\hline
\end{tabular}

TABLE 10: PHYSICAL PROPERTIES OF TABLET FORMULATIONS (L1 TO L9) OF ATENOLOL SUSTAINED RELEASE MATRIX TABLETS

\begin{tabular}{cccccccccc}
\hline Parameters & \multicolumn{10}{c}{ Formulation Codes } \\
\cline { 2 - 10 } & L1 & L2 & L3 & L4 & L5 & L6 & L7 & L8 & L9 \\
\hline Drug content (\%) & 98.37 & 96.86 & 97.09 & 103.48 & 100.81 & 99.18 & 95.11 & 99.41 & 100.34 \\
Hardness (kg/cm ${ }^{3}$ ) & 10.8 & 11.0 & 12.2 & 11.2 & 12.4 & 12.0 & 12.6 & 13.2 & 13.4 \\
Swelling index (\%) & 83.87 & 143.64 & 214.03 & 194.75 & 222.82 & 251.13 & 194.27 & 240.44 & 275.31 \\
Buoyancy lag time (min) & 1.30 & 1.20 & 2.30 & 1.35 & 1.30 & 1.10 & 1.00 & 1.55 & 2.05 \\
\hline
\end{tabular}

TABLE 11: PHYSICAL PROPERTIES OF TABLET FORMULATIONS (I1 TO I9) OF ATENOLOL SUSTAINED RELEASE MATRIX TABLETS

\begin{tabular}{|c|c|c|c|c|c|c|c|c|c|}
\hline \multirow{2}{*}{ Parameters } & \multicolumn{9}{|c|}{ Formulation Codes } \\
\hline & I1 & I2 & I3 & I4 & I5 & I6 & I7 & I8 & I9 \\
\hline Drug content $(\%)$ & 100.46 & 96.74 & 95.46 & 98.83 & 100.81 & 99.53 & 99.18 & 101.27 & 95.11 \\
\hline Hardness $\left(\mathrm{kg} / \mathrm{cm}^{3}\right)$ & 8.8 & 6.6 & 7.2 & 6.4 & 5.8 & 6.6 & 5.6 & 5.2 & 5.6 \\
\hline Swelling index (\%) & 84.32 & 142.43 & 212.46 & 196.97 & 225.25 & 250.25 & 196.14 & 242.14 & 272.5 \\
\hline Buoyancy lag time (min) & 1.45 & 2 & 2.05 & 2.30 & 2.25 & 1.30 & 1.45 & 3 & 3.45 \\
\hline
\end{tabular}

TABLE 12: PHYSICAL PROPERTIES OF TABLET FORMULATIONS (H1 TO H9) OF ATENOLOL SUSTAINED RELEASE MATRIX TABLETS

\begin{tabular}{|c|c|c|c|c|c|c|c|c|c|}
\hline \multirow{2}{*}{ Parameters } & \multicolumn{9}{|c|}{ Formulation Codes } \\
\hline & L1 & L2 & $\mathbf{L 3}$ & L4 & L5 & L6 & L7 & L8 & L9 \\
\hline Drug content $(\%)$ & 96.62 & 99.18 & 99.53 & 96.62 & 102.44 & 100.11 & 98.02 & 100.23 & 101.39 \\
\hline Hardness $\left(\mathrm{kg} / \mathrm{cm}^{3}\right)$ & 9.4 & 9.8 & 10.2 & 9.6 & 8.0 & 8.4 & 8.6 & 7.8 & 7.6 \\
\hline Swelling index $(\%)$ & 152.59 & 175.81 & 184.04 & 183.87 & 200.25 & 222.16 & 202.48 & 225.75 & 227.82 \\
\hline Buoyancy lag time (min) & 0.50 & 1 & $1-1.5$ & 1 & 0.45 & 1.30 & 5 & 1.40 & 2 \\
\hline
\end{tabular}

TABLE 13: KINETICS OF OPTIMIZED FORMULATIONS FROM ALL THE FACTORIAL BATCHES

\begin{tabular}{cccccccccc}
\hline \multicolumn{1}{c}{ S. } & \multirow{2}{*}{ Model } & \multicolumn{2}{c}{$\mathbf{F 6}$} & \multicolumn{2}{c}{$\mathbf{L 6}$} & \multicolumn{2}{c}{$\mathbf{I 2}$} & \multicolumn{1}{c}{$\mathbf{H 4}$} \\
\cline { 2 - 9 } no. & & $\mathbf{R}^{\mathbf{2}}$ & $\mathbf{k}$ & $\mathbf{R}^{\mathbf{2}}$ & $\mathbf{k}$ & $\mathbf{R}^{2}$ & $\mathbf{k}$ & $\mathbf{R}^{2}$ & $\mathbf{k}$ \\
\hline 1 & Zero order & 0.9803 & 11.4925 & 0.859 & 9.4253 & 0.887 & 9.4595 & 0.650 & 0.0077 \\
2 & First order & 0.6791 & -0.3711 & 0.962 & -0.1391 & 0.973 & -0.1407 & 0.650 & -0.0001 \\
3 & Higuchi matrix & 0.9184 & 26.8997 & 0.994 & 22,931 & 0.993 & 22.932 & 0.962 & -0.0191 \\
4 & Hixson and Crowell method & 0.9339 & 18.1338 & 0.984 & 25.783 & 0.979 & 24.783 & 0.982 & 0.0270 \\
5 & Korsemeyer and Peppas model & 0.8400 & -0.0688 & 0.937 & -0.0404 & 0.954 & -0.0407 & 0.650 & 0.000 \\
\hline
\end{tabular}

Stability Studies: ${ }^{11-12}$ The optimized formulations were subjected to stability at $25{ }^{0} \mathrm{C} \pm 2{ }^{0} \mathrm{C} / 60 \% \pm$ $5 \% \mathrm{RH}, 30{ }^{0} \mathrm{C} \pm 2{ }^{0} \mathrm{C} / 65 \% \pm 5 \% \mathrm{RH}$ and $40{ }^{0} \mathrm{C} \pm 2$ ${ }^{0} \mathrm{C} / 75 \% \pm 5 \% \mathrm{RH}$ for period of 90 days. The tablet samples were then analysed for physical characteristics and drug release profile. Following table states the stability study results. 
Characterization of Powder Properties: A flow property plays an important role in tablet formulations because in presence of improper flow, it may cause more weight variation. Values of Carr's Index (compressibility) below 25\% gives rise to good flow properties but readings above $25 \%$ indicate poor flow properties. It was found that the compressibility (Carr's index) of the powder were below 25\% and hence they exhibit good flow characteristics. The Carr's index of the powder was in the range of 6.5 to 24.2 , which indicate a good flow property of the powders. The parameter of angle of repose of all the powder blends was obtained within the range of $20-30{ }^{\circ} \mathrm{C}$.

\section{Evaluation of Tablets:}

Physical parameters: The punches that are used to compress the tablets were $8 \mathrm{~mm}$, spherical shaped. The shape and size of the tablets were found to be within the limit. The hardness of the tablets was found to be in the range of 6.4 to $10.1 \mathrm{~kg} / \mathrm{cm}^{2}$. It was within the range of monograph specification. The thickness of tablets was found to be in the range of 1.90 to $1.94 \mathrm{~mm}$. The friability of the tablets was found to be less than $1 \%$ and thus, it was within the range of standard specification.

In-vitro release studies: ${ }^{12-17}$ In-vitro dissolution study (USP Paddle Method) was performed in phosphate buffer $\mathrm{pH} 7.4$ as the dissolution medium. The $\%$ drug release of all the tablet formulations was calculated using the PCP disso software and the drug release pattern was carried out. The cumulative $\%$ drug release patterns of all the formulations are shown in Tables 15 - 18. The concentrations of the solutions are calculated by using the standard regression equation.

\section{TABLE 14: STABILITY STUDY RESULTS}

\begin{tabular}{cccc}
\hline Test & \multicolumn{3}{c}{ Aluminium foil } \\
\cline { 2 - 4 } & Initial & $\mathbf{3 M ~ 2 5}{ }^{\circ} \mathbf{C} / \mathbf{6 0} \% \mathbf{R H}$ & $\mathbf{3 M} \mathbf{4 0}{ }^{\circ} \mathbf{C} / \mathbf{7 5} \% \mathbf{R H}$ \\
\hline Appearance & Complies & Complies & Complies \\
White, round uncoated tablets & & & \\
Assay & $96.9 \%$ & $99.2 \%$ & \\
95-105.0\% of labeled amount & & & $93.3 \%$ \\
Dissolution & $92.0 \%$ & $95.0 \%$ & $98.0 \%$ \\
NLT 85\% (Q) & $91.0 \%$ & $97.0 \%$ & $97.0 \%$ \\
of labelled amount in & $94.0 \%$ & $98.0 \%$ & $92.0 \%$ \\
45 minutes & $90.0 \%$ & $98.0 \%$ & $96.0 \%$ \\
& $93.0 \%$ & $97.0 \%$ & $98.0 \%$ \\
Related Substance & $92.0 \%$ & $96.0 \%$ & 0.013 \\
(i) Impurity F NMT 0.20\% & 0.01 & 0.023 & 0.034 \\
(ii)Any other Known Impurity & & & \\
NMT 0.20\% & 0.025 & 0.046 & 0.059 \\
(iii) Single Maximum Unknown & 0.021 & 0.057 & 0.094 \\
Impurity: NMT 0.10\% & & & 0.103 \\
(iv) Total Impurities NMT 0.50\% & 0.12 & & \\
\hline
\end{tabular}

TABLE 15: CUMULATIVE \% DRUG RELEASE PATTERN (F1 TO F9)

\begin{tabular}{cccccccccc}
\hline Time & \multicolumn{8}{c}{ Formulation Codes } \\
(hrs) & F1 & F2 & F3 & F4 & F5 & F6 & F7 & F8 & F9 \\
\hline 1 & 21.42 & 20.07 & 18.91 & 16.79 & 22.58 & 23.45 & 21.52 & 20.07 & 19.59 \\
2 & 26.63 & 25.76 & 24.12 & 23.54 & 23.74 & 22.29 & 26.93 & 24.71 & 25.67 \\
3 & 31.85 & 29.63 & 29.05 & 32.62 & 28.95 & 32.23 & 33.59 & 29.24 & 30.69 \\
4 & 41.40 & 43.72 & 41.31 & 38.21 & 40.34 & 41.79 & 37.35 & 40.34 & 39.38 \\
5 & 51.54 & 49.03 & 48.06 & 45.17 & 50.77 & 55.79 & 49.03 & 51.23 & 48.84 \\
6 & 57.52 & 51.25 & 55.40 & 56.75 & 57.72 & 60.61 & 57.72 & 60.42 & 53.36 \\
7 & 98.21 & 70.84 & 77.99 & 86.67 & 87.16 & 78.47 & 84.74 & 76.06 & 64.47 \\
8 & - & 99.00 & 96.28 & 97.89 & 98.07 & 99.02 & 100.07 & 96 & 98.06 \\
9 & - & - & - & - & - & - & - & - & - \\
10 & - & - & - & - & - & - & - & - & - \\
\hline
\end{tabular}


From Table 15, it was observed that, after $10 \mathrm{~s}$ of dissolution study of all the formulations shows \% drug release in the range of $96-100.07 \%$.The drug release Pattern is graphically represented in Fig. 1. From the drug release profile, it was observed that formulations F1 - F3 shows comparatively more drug release as compared to other formulation.

The drug release pattern shows that the \% drug release after $12 \mathrm{hrs}$ was found within the range of $76.93-91.46 \%$. The graphical representation of the drug release profile is given in Fig. 2. From the drug release pattern, it was observed that the formulation L4 shows good drug release within 12 h. The drug release pattern shows that the $\%$ drug release after $12 \mathrm{hrs}$ was found within the range of
$89.67-108.49 \%$. The graphical representation of the drug release profile is given in Fig. 3. From the drug release pattern, it was observed that the formulation L4 shows good drug release within 10 h.

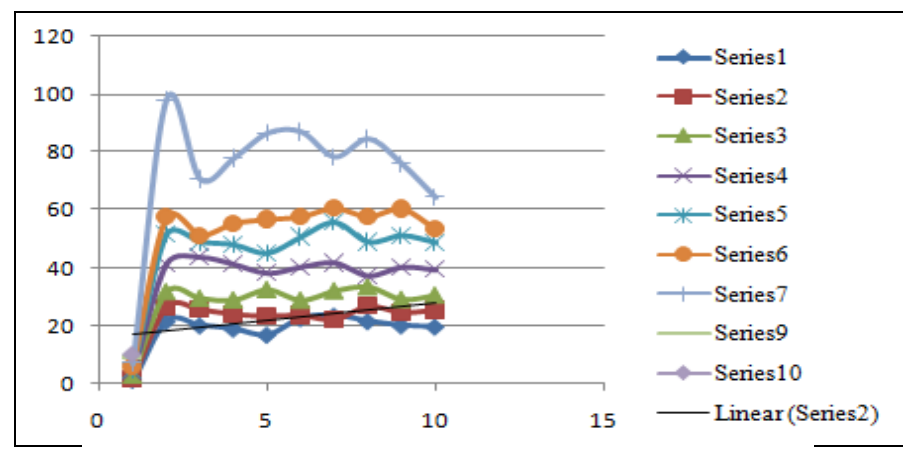

FIG. 1: DISSOLUTION PROFILE OF F1 TO F9

TABLE 16: CUMULATIVE \% DRUG RELEASE PATTERN (L1 TO L9)

\begin{tabular}{|c|c|c|c|c|c|c|c|c|c|}
\hline $\begin{array}{c}\text { Time } \\
\text { (h) }\end{array}$ & \multicolumn{9}{|c|}{ Formulation Codes } \\
\hline 2 & 26.64 & 25.00 & 24.57 & 25.67 & 25.67 & 36.96 & 21.13 & 21.91 & 21.52 \\
\hline 4 & 48.16 & 44.01 & 41.11 & 41.11 & 35.32 & 41.79 & 39.96 & 40.83 & 39.96 \\
\hline 5 & 53.18 & 50.19 & 48.16 & 43.14 & 40.54 & 50.96 & 40.92 & 45.94 & 47.87 \\
\hline 6 & 58.59 & 55.59 & 50.67 & 48.84 & 48.26 & 54.82 & 49.98 & 50.48 & 50.77 \\
\hline 9 & 84.36 & 62.54 & 65.25 & 64.67 & 67.18 & 69.40 & 66.31 & 64.47 & 60.42 \\
\hline 10 & 90.15 & 69.30 & 71.81 & 69.30 & 70.07 & 72.58 & 70.75 & 68.53 & 67.47 \\
\hline 11 & 90.15 & 80.50 & 79.44 & 77.22 & 76.15 & 76.83 & 73.93 & 72.77 & 71.81 \\
\hline 12 & 91.46 & 86.87 & 88.22 & 80.50 & 79.44 & 77.22 & 78.95 & 77.50 & 76.93 \\
\hline
\end{tabular}

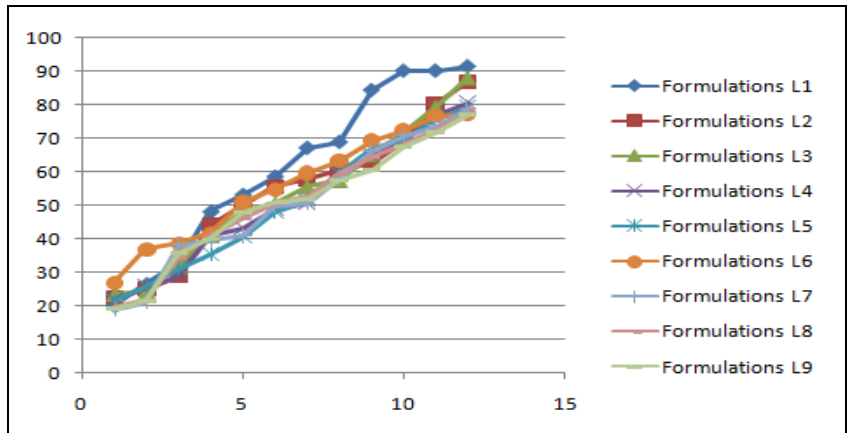

FIG. 2: DISSOLUTION PROFILE OF L1 TO L9

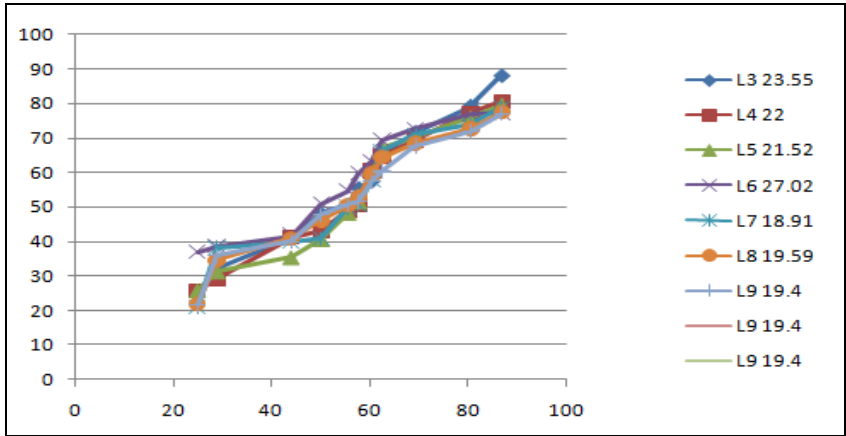

FIG. 3: DISSOLUTION PROFILE OF I1 TO I9

TABLE 17: CUMULATIVE \% DRUG RELEASE PATTERN (I1 TO I9)

\begin{tabular}{|c|c|c|c|c|c|c|c|c|c|}
\hline \multirow{2}{*}{$\begin{array}{c}\text { Time } \\
\text { (h) }\end{array}$} & \multicolumn{9}{|c|}{ Formulation Codes } \\
\hline & I1 & I2 & I3 & I4 & I5 & I6 & I7 & I8 & I9 \\
\hline 1 & 21.52 & 23.71 & 24.51 & 22.49 & 21.52 & 25.09 & 25.19 & 21.13 & 21.62 \\
\hline 2 & 28.76 & 31.21 & 26.44 & 25.38 & 26.46 & 36.58 & 36.38 & 22.87 & 22.49 \\
\hline 3 & 34.16 & 37.35 & 34.16 & 31.17 & 36.00 & 38.51 & 39.54 & 34.65 & 26.64 \\
\hline 4 & 46.62 & 40.83 & 41.21 & 40.44 & 40.83 & 42.37 & 43.87 & 40.44 & 32.72 \\
\hline 5 & 55.50 & 50.03 & 53.37 & 43.72 & 52.99 & 50.09 & 50.99 & 47.97 & 41.79 \\
\hline 6 & 60.03 & 56.08 & 56.66 & 50.09 & 56.37 & 54.63 & 55.12 & 53.08 & 47.00 \\
\hline 7 & 66.31 & 59.74 & 59.74 & 53.95 & 65.16 & 60.13 & 60.43 & 57.62 & 55.59 \\
\hline 8 & 75.77 & 64.28 & 64.28 & 65.57 & 66.31 & 66.21 & 69.31 & 67.56 & 55.59 \\
\hline 9 & 86.58 & 72.77 & 72.77 & 66.31 & 70.75 & 71.23 & 74.28 & 74.90 & 64.96 \\
\hline 10 & 88.89 & 80.59 & 80.59 & 69.98 & 76.15 & 85.61 & 85.64 & 85.61 & 73.06 \\
\hline 11 & 95.17 & 89.18 & 85.32 & 85.52 & 84.74 & 95.46 & 98.41 & 94.20 & 79.05 \\
\hline 12 & 99.03 & 108.39 & 107.25 & 96.81 & 96.13 & 115.73 & 110.43 & 108.49 & 89.67 \\
\hline
\end{tabular}


TABLE 18: CUMULATIVE \% DRUG RELEASE PATTERN (H1 TO H9)

\begin{tabular}{|c|c|c|c|c|c|c|c|c|c|}
\hline \multirow{2}{*}{$\begin{array}{l}\text { Time } \\
\text { (h) }\end{array}$} & \multicolumn{9}{|c|}{ Formulation Codes } \\
\hline & H1 & H2 & H3 & H4 & H5 & H6 & H7 & H8 & H9 \\
\hline 1 & 26.64 & 29.53 & 30.79 & 27.70 & 32.14 & 21.13 & 27.60 & 28.95 & 32.14 \\
\hline 2 & 30.89 & 33.49 & 37.35 & 31.17 & 35.42 & 26.64 & 31.27 & 32.72 & 33.59 \\
\hline 3 & 36.29 & 38.22 & 40.34 & 34.65 & 37.64 & 28.36 & 36.29 & 35.42 & 36.58 \\
\hline 4 & 40.92 & 40.83 & 44.69 & 37.35 & 38.57 & 31.17 & 39.18 & 38.22 & 41.40 \\
\hline 5 & 48.06 & 45.94 & 48.97 & 40.44 & 41.98 & 35.31 & 41.89 & 41.79 & 43.72 \\
\hline 6 & 60.23 & 50.09 & 50.48 & 44.69 & 44.98 & 37.16 & 46.04 & 45.94 & 47.00 \\
\hline 7 & 65.25 & 57.62 & 57.52 & 47.00 & 48.35 & 40.44 & 49.03 & 48.35 & 50.48 \\
\hline 8 & 71.33 & 61.35 & 61.29 & 48.74 & 55.59 & 44.98 & 51,54 & 51.83 & 54.63 \\
\hline 9 & 76.33 & 67.37 & 67.56 & 56.66 & 58.59 & 47.97 & 54.72 & 56.56 & 56.66 \\
\hline 10 & 79.05 & 70.55 & 69.78 & 61.29 & 60.42 & 50.77 & 57.52 & 59.74 & 60.23 \\
\hline 11 & 85.42 & 75.86 & 74.90 & 65.05 & 66.11 & 51.54 & 60.23 & 65.25 & 63.32 \\
\hline 12 & 88.70 & 79.24 & 79.3 & 69.40 & 70.84 & 56.66 & 64.28 & 68.23 & 67.08 \\
\hline
\end{tabular}

The drug release pattern shows that the \% drug release after $12 \mathrm{hrs}$ was found within the range of $56.66-88.70 \%$. The graphical representation of the drug release profile is given in Fig. 4. From the drug release pattern, it was observed that the formulation $\mathrm{H} 4$ shows good drug release within 12 h.

Release Experiments: The release pattern of all the formulations was calculated using PCP disso V3 software. All the formulations were fitted for zero order release, first order release, Higuchi matrix model, Hixson and crowell powder dissolution model and korsemeyer-peppas model. The equations for all the models are shown in the Table 19 - 22.

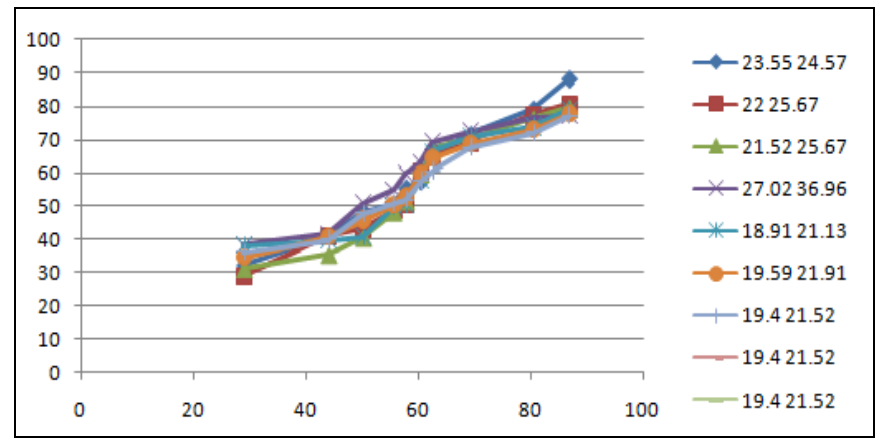

FIG. 4: DISSOLUTION PROFILE OF H1 TO H9

TABLE 19: BEST FITTING MODELS FOR FORMULATIONS F1 TO F9

\begin{tabular}{cc}
\hline Formulation & Best fitting model \\
\hline F1 & Zero order \\
F2 & Zero order \\
F3 & Zero order \\
F4 & Zero order \\
F5 & Zero order \\
F6 & Zero order \\
F7 & Zero order \\
F8 & Zero order \\
F9 & Zero order \\
\hline
\end{tabular}

TABLE 20: BEST FITTING MODELS FOR FORMULATIONS L1 TO L9

\begin{tabular}{cc}
\hline Formulation & Best fitting model \\
\hline L1 & $1^{\text {st }}$ order \\
L2 & Matrix \\
L3 & Matrix \\
L4 & Matrix \\
L5 & Matrix \\
L6 & Matrix \\
L7 & Matrix \\
L8 & Matrix \\
L9 & Matrix \\
\hline
\end{tabular}

TABLE 21: BEST FITTING MODELS FOR FORMULATIONS I1 TO I9

\begin{tabular}{cc}
\hline Formulation & Best fitting model \\
\hline I1 & Hixon Crowel \\
I2 & Matrix \\
I3 & Matrix \\
I4 & Matrix \\
I5 & $1^{\text {st }}$ order \\
I6 & Matrix \\
I7 & Matrix \\
I8 & $1^{\text {st }}$ order \\
I9 & Matrix \\
\hline
\end{tabular}

TABLE 22: BEST FITTING MODELS FOR FORMULATIONS H1 TO H9

\begin{tabular}{cc}
\hline Formulation & Best fitting model \\
\hline H1 & Matrix \\
H2 & Matrix \\
H3 & Korsemeyer Peppas \\
H4 & Korsemeyer Peppas \\
H5 & Korsemeyer Peppas \\
H6 & Korsemeyer Peppas \\
H7 & Korsemeyer Peppas \\
H8 & Korsemeyer Peppas \\
H9 & Korsemeyer Peppas \\
\hline
\end{tabular}

FTIR Study: FTIR spectrums of the drug and excipients were taken and it shows no interaction among each other. 


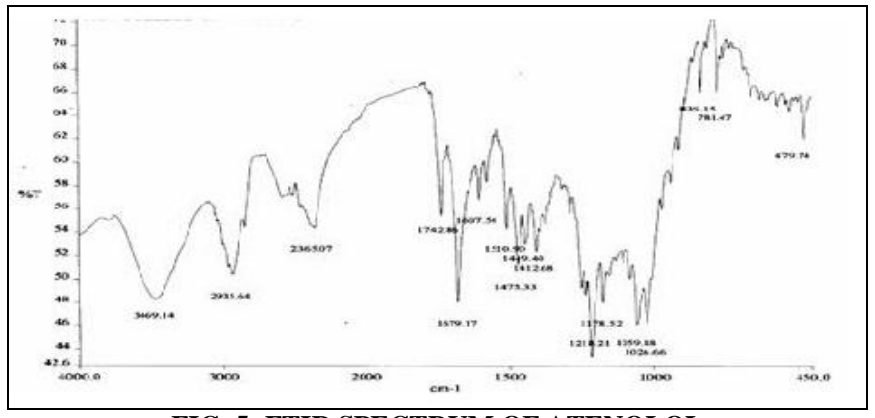

FIG. 5: FTIR SPECTRUM OF ATENOLOL

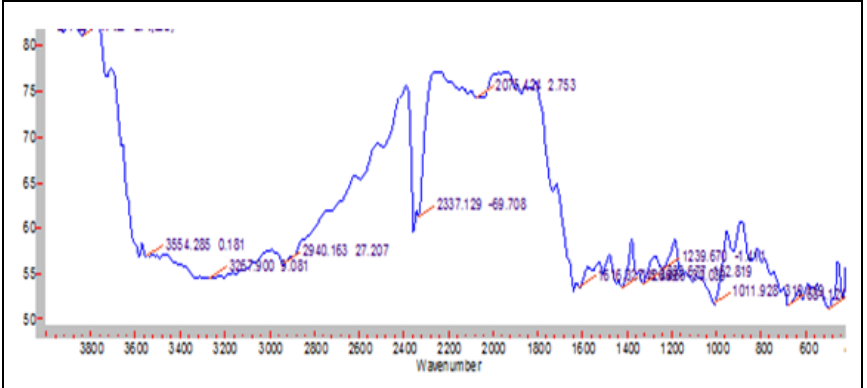

FIG. 6: FTIR SPECTRUM OF OPTIMIZED FORMULATION F6

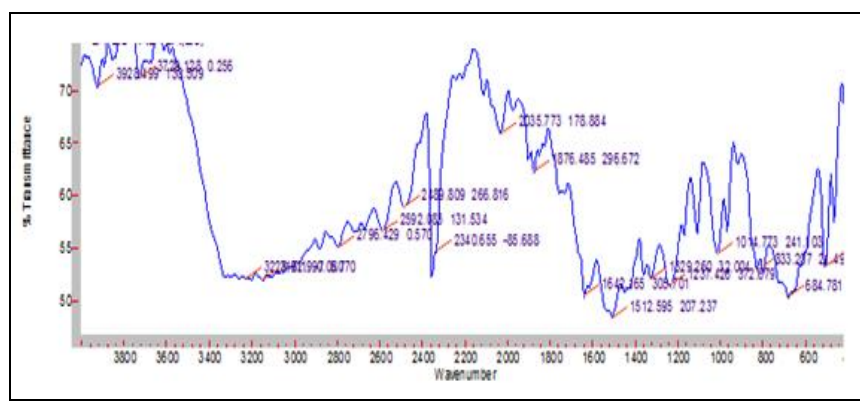

FIG. 8: FTIR SPECTRUM OF OPTIMIZED FORMULATION I2

Contact Angle Measurement: ${ }^{19}$ The drop of phenolphthalein was placed on sintered and unsintered tablet. The droplet spreads immediately on the unsintered tablet due to more porosity while the droplet remains intact on the surface of sintered tablet due to less porosity. The effect of sintering on the wettability of the tablet surfaces was established by taking the photographs of the tablet surfaces on which drop of colored water was placed. Contact angle is indicative of the wettability of the tablet surface.

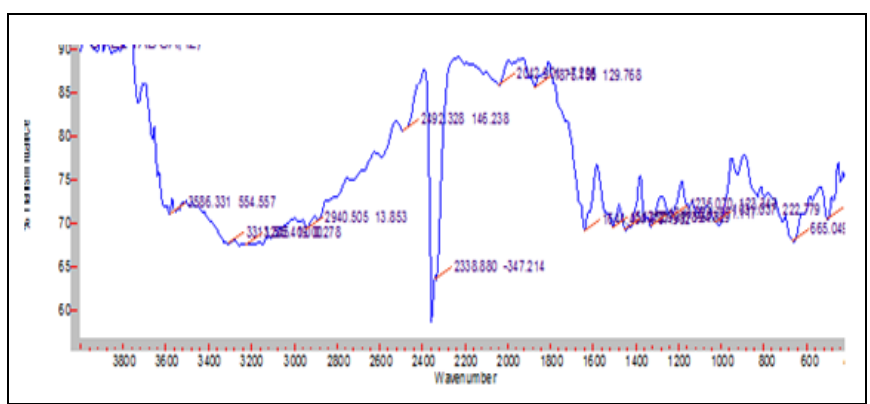

FIG. 7: FTIR SPECTRUM OF OPTIMIZED FORMULATION L2

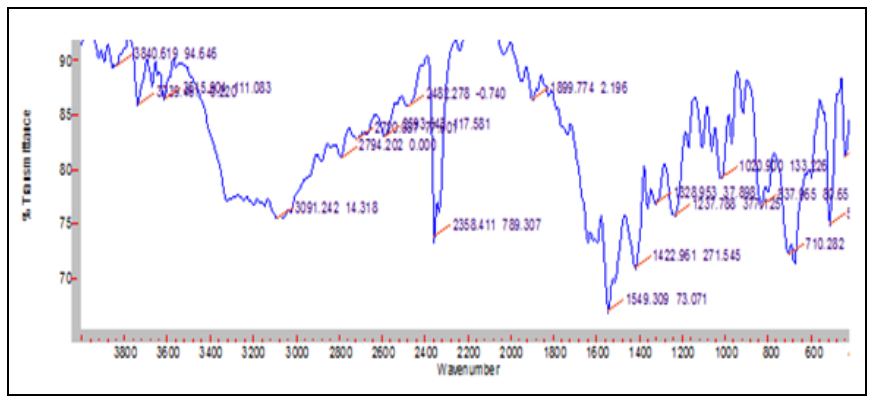

FIG. 9: FTIR SPECTRUM OF OPTIMIZED FORMULATION H4

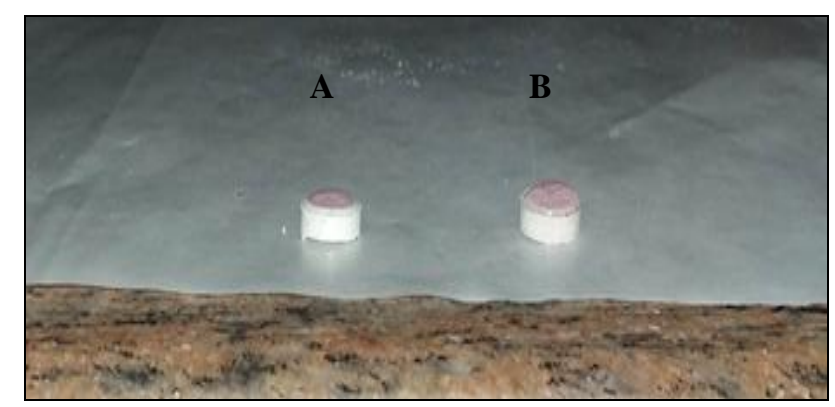

FIG. 10: (A) DROPLET IS SEEN TO BE STILL ON THE SINTERED TABLET (B) DROPLET IS SEEN TO BE IMMERSED IN THE UNSINTERED TABLET

Powder XRD Study: Following figure shows pXRD graph of Atenolol in mixture.

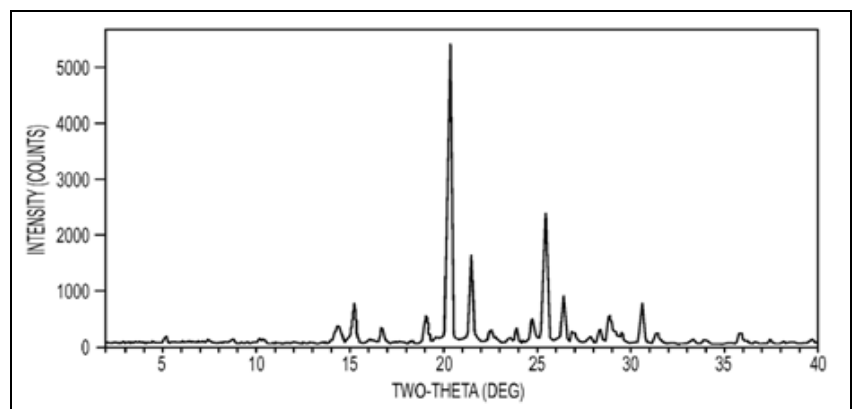

FIG. 11: pXRD GRAPH OF ATENOLOL IN MIXTURE

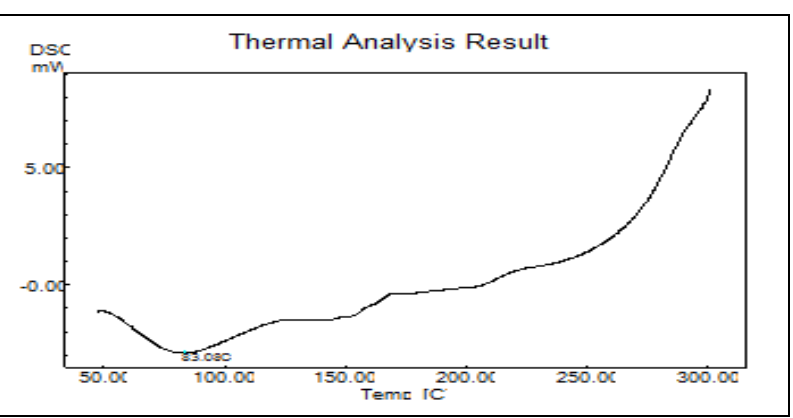

FIG. 12: DSC GRAPH OF ATENOLOL IN MIXTURE 
DSC Study: Following graph shows result of differential scanning calorimetry.

\section{Scanning Electron Microscopy (SEM) Study:}
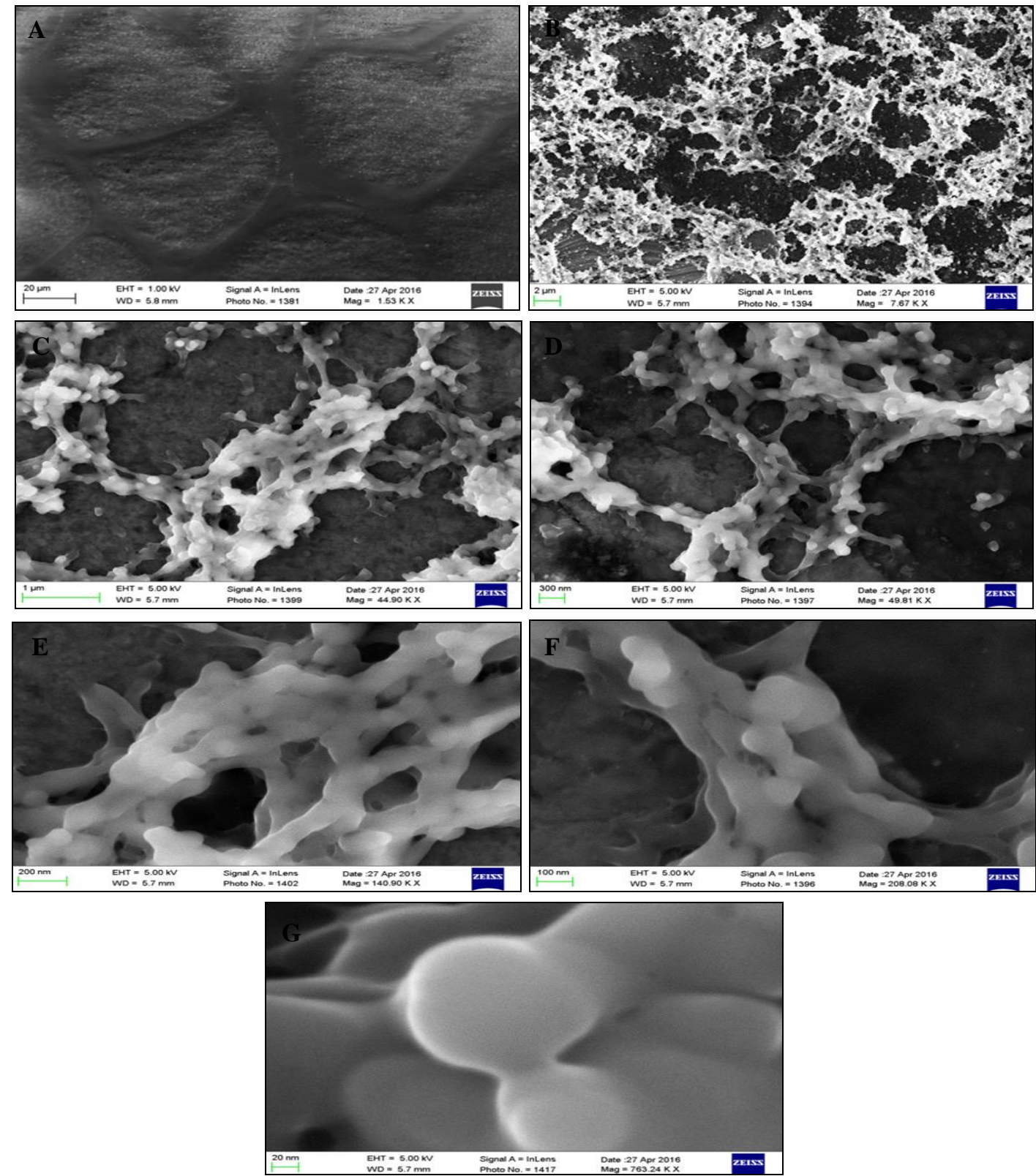

FIG. 13: SEM OF ATENOLOL WITH INCREASING SINTERING TEMPERATURE AND SINTERING TIME SEQUENCING FROM (A) TO (G)

CONCLUSION: The aim of the study was to formulate a stable as well as robust dosage form. The in-vitro dissolution of the test formulation is comparable with innovator reference formulation in all media. Matrix tablet of atenolol can be prepared successfully by wet granulation method using tragacanth, Eudragit polymers as retardant and by using lactose as filler. Sintering technique is optimized for the preparation of robust, effective SR tablet of atenolol. This technique describes the mechanism of strengthening the mechanical properties of consolidated pharmaceutical powders at elevated temperatures, for solid bond formation during tablet compression. In present study sintering was carried out at $60{ }^{\circ} \mathrm{C}, 70{ }^{\circ} \mathrm{C}, 80{ }^{\circ} \mathrm{C}$ for $1.5,3,4.5 \mathrm{~h}$. Coalescence of Eudragit particles is influenced by minimum film forming temperature.

Exposure to such kind of temperature makes the structural changes in the tablet compacts that resulted in matrix tablet structure. On sintering it was found that F6, L2, I2 and H4 gave better results compared to other formulations. The aim was to release the loading dose $111 \mathrm{mg}$ in first $2 \mathrm{~h}$ 
and release the remaining amount in next $22 \mathrm{~h}$ and therefore thermal sintering was optimized.

On applying various models on the dissolution study release all the formulations showed matrix diffusion model. As tablets were sintered by thermal exposure, the sustained release of drug may be due to the chemical interaction with polymer. The hydrophobic properties of surfaces after sintering were increased. This correlated well with the SEM micrographs. The lower wettability is responsible for retardation of drug release due to less penetration of dissolution medium in matrix tablet.

FTIR spectrums and DSC graph of the drug and excipients were taken and it shows no interaction among each other.

The XRD study results revels that the powder characteristics were same as that of standard one after stability. The final prepared tablets were charged for stability study, samples were withdrawn and tablet showed no change in physical appearance, related substance, assay and drug release. Thus stability results prove that the formulation was stable at accelerated condition.

The final prepared tablets were charged for stability study, samples were withdrawn and tablet showed no change in physical appearance, related substance, assay and drug release. Thus stability results prove that the formulation was stable at accelerated condition. During sintering of tablets, polymer particles transform from glossy state to rubbery state and redistributed to entangle the drug particles, leading to drug release retardation.

ACKNOWLEDGEMENT: The authors would like to thank the Management, MAEER's, Management, Principal Maharashtra Institute of Pharmacy, Pune - India for providing laboratory facilities and supporting this research work

CONFLICT OF INTEREST: The authors declare that there is no conflict of interest in publication of this paper.

\section{REFERENCES:}

1. Turkoglu M and Adel S: Tablet dosage form. In: Florence AT, Siepmann J, editors. Morden pharmaceutics: basic principles and systems. New York, Information healthcare, Edition $5^{\text {th }}$, 2009; 481-498.

2. Thakkar $\mathrm{N}$ and Gupta MM: International Journal of Pharmaceutical Research and Allied Sci, 2012; 1(1): 1-13.

3. Banker GS, Anderson NR, In Lachman L, Lieberman HA and Kanig JL: The Theory and Practice of Industrial Pharmacy. Varghese Publishing House, Mumbai, Edition $3^{\text {rd }}, 2009$; $293-$ 345.

4. Lachman L, Lieberman HA and Kanig JL: The Theory and Practice of Industrial Pharmacy. Tablets; Varghese publishing house, Bombay, Edition $3^{\text {rd }}$, 2010; 47-66, 294, 336, 413.

5. Sweetman SC: Martindale: The complete drug reference. Pharmaceutical Press. London and Chicago Edition $34^{\text {th }}$, 2005; 900-1.

6. Sweetman SC: Martindale-The complete drug reference; Drug monographs, Pharmaceutical press, London, Vol. A, Edition $37^{\text {th }}, 1271$.

7. Polshettiwar S, Hajare RA, et al:: Formulation and evaluation of gastroretentive Drug delivery system of carevedilol phosphate by Sintering technology. World Journal of Pharmacy and Pharmaceutical Sciences (WJPS), 2016; 5(4): 959-978.

8. Patel DM, Patel BK, Patel HA and Patel CN: Design of controlled release non-erodible polymeric matrix tablet using microwave oven-assisted sintering technique. J Young Pharmacists 2011; 3: 176-80.

9. Kadam VD and Gattani SG: Asian Journal of Pharmaceuticals and Clinical Reserach, 2009; 3(2): 39-43.

10. Rao MR, Shivpuje S, Godbole R and Shirsath C: Design and evaluation of sustained release matrix tablets using sintering technique, IJPPS, 2016; 8(2): 115-121.

11. Azeem $S$ and Sharma S; International Journal of Biopharm. And toxicol. Res; 2011; 1(1): 24-46.

12. Tripp JH, Sandhu BK, Candy DCA and Harries JT; Studies on Mechanism of Action; Gut; 1981; 22: 658-662.

13. Parvathi $\mathrm{M}$, Prathyusha $\mathrm{P}$ and Raveendrareddy J: Formulation and evaluation of sintered matrix tablets of metformin hydrochloride and its comparision over unsintered matrix tablets, RJRPC, 2013; 3(3): 521-529.

14. Paul Y, Kumar M and Singh B: Formulation and in-vitro evaluation of gastroretentive drug delivery system of cefixime trihydrate. Int Jou of Drug Development and Research 2011.

15. Mohanty $\mathrm{C}$ and Subrahmanyam KV: Effect of sintering condition on physico-chemical parameters and drug release characteristics from polymeric matrix tablet of atenolol for controlled release. Int J Pharm Sci Res 2017; 8(9): 3758-67. doi: 10.13040/IJPSR.0975-8232.8(9).3758-67.

16. ICH guideline for bioequivalence studies for different strengths of oral solid dosage forms. 2008; 3-12. Jorgensen AC: Increasing process understanding of wet granulation by spectroscopic methods and dimension reduction tools; Faculty of Pharmacy of the University of Helsinki, 2004.

17. Pandey S: Optimizing fast dissolving dosage form of diclofenac sodium by rapidly disintegrating agents. Indian Journal of Pharmaceutical Sciences, 2003; 65(2): 197-201.

18. Polshettiwar S, Patil C, Battuwar A and Hajare R: Formulation and evaluation of sintered matrix tablets of caffiene and its comparision over unsintered matrix tablets. IAJPR, 2016; 6: 4843-4858.

\section{How to cite this article:}

Polshettiwar S and Hajare R: Design of controlled release non-erodible polymeric atenolol matrix tablet using microwave oven-assisted sintering technique. Int J Pharm Sci \& Res 2018; 9(8): 3388-97. doi: 10.13040/IJPSR.0975-8232.9(8).3388-97.

All @ 2013 are reserved by International Journal of Pharmaceutical Sciences and Research. This Journal licensed under a Creative Commons Attribution-NonCommercial-ShareAlike 3.0 Unported License. This article can be downloaded to ANDROID OS based mobile. Scan QR Code using Code/Bar Scanner from your mobile. (Scanners are available on Google Playstore) 